\title{
Parâmetros de dispersão de ar em células mecânicas de flotação em usinas brasileiras
}

\author{
Air dispersion parameters in mechanical \\ flotation cells of industrial Brazilian plants
}

Ricardo José Montesinos Ojeda

Mestrando em Engenharia Mineral da

Escola Politécnica da USP.

Departamento de Engenharia de Minas

e de Petróleo, Escola Politécnica,

Universidade de São Paulo, São Paulo, SP, Brasil.

ricardo.montesinos@usp.br

Odair Alves de Lima

Pesquisador, PhD,

AkzoNobel SC, Brasil.

odair.lima@akzonobel.com

\section{Massimiliano Zanin}

Ian Wark Research Institute,

University of South Australia,

Mawson Lakes, Austrália.

massimiliano.zanin@unisa.edu.au

\section{Laurindo de Salles Leal Filho}

Professor Titular da Escola Politécnica da USP.

Departamento de Engenharia de Minas

e de Petróleo, Escola Politécnica,

Universidade de São Paulo, São Paulo, SP, Brasil. lauleal@usp.br

\section{Resumo}

O objetivo desse trabalho é apresentar e discutir a determinação dos parâmetros de dispersão de ar em células mecânicas de flotação, em circuitos industriais brasileiros. Foram investigados os circuitos rougher e scavenger das usinas RPM, Taquari-Vassouras, Copebras e Fosfértil-Catalão (atual Vale Fertilizantes). Nas usinas RPM e Taquari-Vassouras, foram determinados a velocidade superficial do ar $\left(\mathrm{J}_{\mathrm{g}}\right)$, o hold-up do ar $\left(\varepsilon_{\mathrm{g}}\right)$, o diâmetro médio de bolhas $\left(\mathrm{d}_{32}\right)$ e o fluxo de área superficial de bolhas $\left(\mathrm{S}_{\mathrm{b}}\right)$. Para as outras usinas, foram determinados somente o $\mathrm{J}_{\mathrm{g}}$ e $\varepsilon_{\mathrm{g}}$. Os resultados mostraram que, com exceção da RPM, todos os circuitos investigados apresentaram baixos valores para os parâmetros de dispersão do ar. Taquari-Vassouras apresentou os mais baixos valores médios para $\mathrm{J}_{\mathrm{g}}$ e $\varepsilon_{\mathrm{g}}$, respectivamente, $0,05 \mathrm{~cm} / \mathrm{s}$ e $5 \%$. Os maiores valores de $\mathrm{J}_{\mathrm{g}}$ foram determinados no circuito rougher da Copebras, $1,17 \mathrm{~cm} / \mathrm{s}$, ao passo que a RPM apresentou os maiores valores para o hold-up e tamanho de bolha, respectivamente, $31 \%$ e $2,61 \mathrm{~mm}$. Os valores obtidos, para $S_{b}$, situaram-se abaixo daqueles reportados na literatura.

Palavras-chave: Velocidade superficial do ar, hold-up do ar, tamanho de bolhas, célula de flotação.

\section{Abstract}

The aim of this paper is to present and discuss the air dispersion parameters determined for industrial Brazilian flotation circuits. The rougher and scavenger circuits of RPM, Taquari-Vassouras, Copebras and Fosfertil-Catalão (today Vale Fertilizantes) were investigated. For RPM and Taquari-Vassouras, the superficial gas velocity $(\mathrm{Jg})$, gas hold-up $\left(\varepsilon_{g}\right)$, bubble size diameter $\left(d_{32}\right)$ and superficial area bubble flux $\left(S_{b}\right)$ were measured, whereas only the $J_{g}$ and $\varepsilon_{g}$ were measured in the other circuits. The results pointed out that, except for RPM, all the circuits presented very low values for the air dispersion parameters. Taquari-Vassouras presented the lowest values for average $J_{g}$ and gas hold-up: $0.05 \mathrm{~cm} / \mathrm{s}$ and $5 \%$, respectively. The highest $J_{g}$ value was presented for the rougher circuit of Copebras, $1.17 \mathrm{~cm} / \mathrm{s}$, while RPM presented the highest values for $\varepsilon_{g}$ and bubble size, respectively $31 \%$ and $2.61 \mathrm{~mm}$. In the circuits where the calculation of the $S_{b}$ parameter was possible, the results obtained lay in a range lower than those reported in literature.

Keywords: Superficial gas velocity, gas hold-up, bubble size, flotation cell. 


\section{Introdução}

No âmbito da Engenharia Mineral, a flotação por espumas, ou, simplesmente, flotação, é uma operação unitária amplamente utilizada na concentração de partículas sólidas, explotando as diferenças nas suas propriedades de superfície. De acordo com Lima (2009), para que o processo de flotação apresente adequada seletividade e recuperação, é necessária a conjunção adequada de fatores físico-químicos e hidrodinâmicos. Enquanto os primeiros estão associados a características tecnológicas/mineralógicas do minério, à natureza e à dosagem dos reagentes, à qualidade da água e ao $\mathrm{pH}$ da suspensão, os fatores hidrodinâmicos estão relacionados com as células de flotação per se (coluna, mecânica ou pneumática), com a geometria e com as condições operacionais (vazão de polpa, vazão de ar, rotação do impelidor, distribuição dos tempos de residência, status da suspensão de sólidos). Quando se ajustam

\section{Velocidade superficial do ar, $J_{g}$}

A velocidade superficial do ar $\left(J_{g}\right)$ é definida como o volume de ar que atravessa a seção transversal da célula por unida-

Gorain et al., (1996) desenvolveram um método para medir o $J_{g}$ em circuitos industriais. Tal método

Devido às diferentes velocidades de ascensão do ar, entre o ponto de coleta

Nessa equação, $\rho_{\mathrm{w}}$ é a massa específica da água aerada, dentro da sonda; $\mathrm{H}_{w}$ é a distância entre a extremidade inferior da sonda e a primeira marca que

\section{Hold-up do ar, $\varepsilon_{\mathrm{g}}$}

O hold-up do ar representa a fração volumétrica ocupada pelo ar em relação ao volume total ocupado pela polpa (líquido+sólido+ar). O hold-up do ar, em células de flotação, geralmente, está com- os fatores físico-químicos, as condições hidrodinâmicas passam a governar $\mathrm{o}$ desempenho do processo. Por muito tempo, a hidrodinâmica dos sistemas de flotação foi relegada a um papel secundário, quando comparada com os parâmetros físico-químicos interfaciais envolvidos na flotação. No entanto, face à crescente quantidade de trabalhos relacionados a essa área de pesquisa, tal cenário tem mudado bastante e, cada vez mais, os fatores hidrodinâmicos são critérios efetivos de projeto e de escalonamento de células de flotação (Weber et al., 1999; Cilek e Yilmazer, 2003; Villanueva et al., 2005). No que tange à hidrodinâmica de circuitos de flotação industriais brasileiros, pouco material tem sido encontrado na literatura especializada, como o trabalho desenvolvido por Lima et al. (2005) e muito pouco se sabe sobre a magnitude ou comportamento dos parâmetros de dispersão de ar em circuitos de flotação

de de tempo e representa uma medida da capacidade de aeração da célula. Tal variável é expressa de acordo com a Eq. (1),

$$
J_{g}=\frac{V_{a r}}{t \cdot A_{c}}
$$

consiste em observar o tempo t decorrido, para que a interface líquidoar percorra uma determinada distân-

$$
\bar{J}_{g}=\frac{L}{t}
$$

(dentro da polpa mineral) e o ponto de medida (dentro do tubo cilíndrico), uma

$$
J_{g}=\bar{J}_{g} \cdot \frac{P_{a t m}+\rho_{p} g H_{p}-\rho_{w} g H_{w}}{P_{a t m}+\rho_{p} g H_{p}}
$$

delimita a distância L percorrida pela interface líquido-ar; $\rho_{\mathrm{p}}$ é a massa específica da polpa aerada; $\mathrm{H}_{\mathrm{p}}$ é a altura da coluna de polpa, entre a interface da brasileiros, de forma que esse trabalho tem, como objetivo, contribuir para o preenchimento dessa lacuna.

Alguns dos dados apresentados, nesse trabalho, são frutos da colaboração científica do PMI/EPUSP com o Instituto de Pesquisas Ian Wark, localizado em Adelaide, Austrália, através do projeto internacional AMIRA P260D, ocasião em que foram caracterizados os parâmetros de dispersão de ar nas usinas de TaquariVassouras (Vale Fertilizantes) e Rio Paracatu Mineração (RPM). Após esse trabalho colaborativo (Weedon et al., 2005), quando houve a transferência de tecnologia para medição desses parâmetros para a USP, foram, também, caracterizadas as usinas de beneficiamento de rocha fosfática Copebrás e Fosfértil (atual Vale-Fertilizantes), ambas em Catalão-GO. Foram determinados a velocidade superficial do ar, o hold-up do ar, o diâmetro médio de bolhas (para alguns casos) e, também, para alguns casos, o fluxo de área superficial de bolhas.

em que $\mathrm{V}_{\mathrm{ar}}$ é o volume de ar que atravessa a célula; $\mathrm{A}_{\mathrm{c}}$ é a área da seção transversal da célula e t representa o tempo.

cia L, dentro de um tubo cilíndrico transparente. Posteriormente, aplicase a Eq. (2).

correção, na Eq. (2), deve ser considerada, de acordo com a Eq. (3).

camada de espuma/polpa e o ponto de coleta das bolhas de ar, e $J_{g}$ representa o valor experimental da velocidade superficial do ar. preendido na faixa de 15-22\% (Deglon et al., 2000; Gorain et al., 1995; Yianatos et al., 2001). No entanto, podem apresentar valores de magnitudes tão baixas quanto $3 \%$ e tão altas quanto 33\% (Deglon et al., 2000; Gorain et al., 1995; Schwarz e Alexander., 2006). Tais variações são devidas a vários fatores, tais como a geometria do tipo de impelidor, como mostra os resultados obtidos por Gorain et al., 
(1995), em que o hold-up do ar variou de $5 \%$ a $19 \%$ de acordo com o tipo de im- pelidor utilizado. O hold-up do ar pode ser calculado de acordo com a Eq. (4), em

$$
\varepsilon_{g}=\frac{V_{a r}}{V_{p}}
$$

que $\mathrm{V}_{\mathrm{ar}}$ é o volume ocupado pelo ar e $\mathrm{V}_{\mathrm{p}}$ é o volume da polpa na célula de flotação.

\section{Tamanho médio de bolhas $\left(d_{32}\right)$ e fluxo superficial de área de bolhas $\left(S_{b}\right)$}

De acordo com a literatura (Ahmed e Jamenson, 1989; Deglon et al., 2000; Grau e Heiskenen, 2005), o tamanho de bolhas é considerado um importante indicador da eficiên- cia da capacidade dispersão de ar de uma célula de flotação. Dada uma distribuição de tamanho de bolhas, o diâmetro médio de Sauter $\left(d_{32}\right)$ é parâmetro, normalmente, utilizado

$$
d_{32}=\frac{\sum_{i=1}^{m} d_{i}^{3}}{\sum_{i=1}^{m} d_{i}^{2}}
$$

Grau e Heiskenen (2005) mostraram que o tamanho médio de bolhas $\left(d_{32}\right)$ é afetado por fatores como rotação do impelidor, vazão de ar e concentra-

A literatura (Gorain et al., 1995; Deglon et al., 2000; Vera, 2002) considera $S_{b}$ um importante parâmetro, que pode ser usado para

\section{Materiais e métodos}

\section{Velocidade superficial do ar}

Os parâmetros de dispersão de ar apresentados e discutidos, nesse trabalho, foram determinados utilizandose sondas desenvolvidas no JKMRC (Julius Kruttschnitt Mineral Research Centre), localizado em Brisbane, Austrália. Para a determinação da velocida- para representar o diâmetro médio de bolhas. Tal parâmetro é definido pela Eq. (5), em que $d_{i}$ representa o diâmetro da esfera de igual volume que a bolha. ção (dosagem) de espumante na célula de flotação. Outra variável de dispersão de ar, derivada das anteriores, é o fluxo de área superficial de bolhas $\left(S_{b}\right)$, que,

$$
S_{b}=6 \cdot \frac{J_{g}}{d_{32}}
$$

avaliar o desempenho de uma célula de flotação, porque incorpora, em uma única variável, não só a eficiência na dispersão de gás (através quando se consideram bolhas esféricas e de tamanho uniforme, ele pode ser definido de acordo com a Eq. (6). do diâmetro médio de bolhas), mas, também, a capacidade de aeração da célula de flotação (através da velocidade superficial do ar). de superficial do ar $\left(J_{g}\right)$, a sonda- $J_{g}$ foi colocada dentro da célula de flotação, na região da polpa, e, através do acionamento de válvulas pneumáticas, foi permitida a entrada de polpa aerada na sonda- $J_{g}$, que, por sua vez, foi, previamente, preenchida com água e, à medi- da que as bolhas de ar se acumulavam na parte superior do tubo cilíndrico, observou-se o deslocamento da interface ar-líquido no interior do tubo. A Figura 1 ilustra a determinação experimental do $J_{g}$, em célula mecânica de flotação.
Figura 1

Medição do $J_{g}$, em célula mecânica de flotação (Weedon et al., 2005).

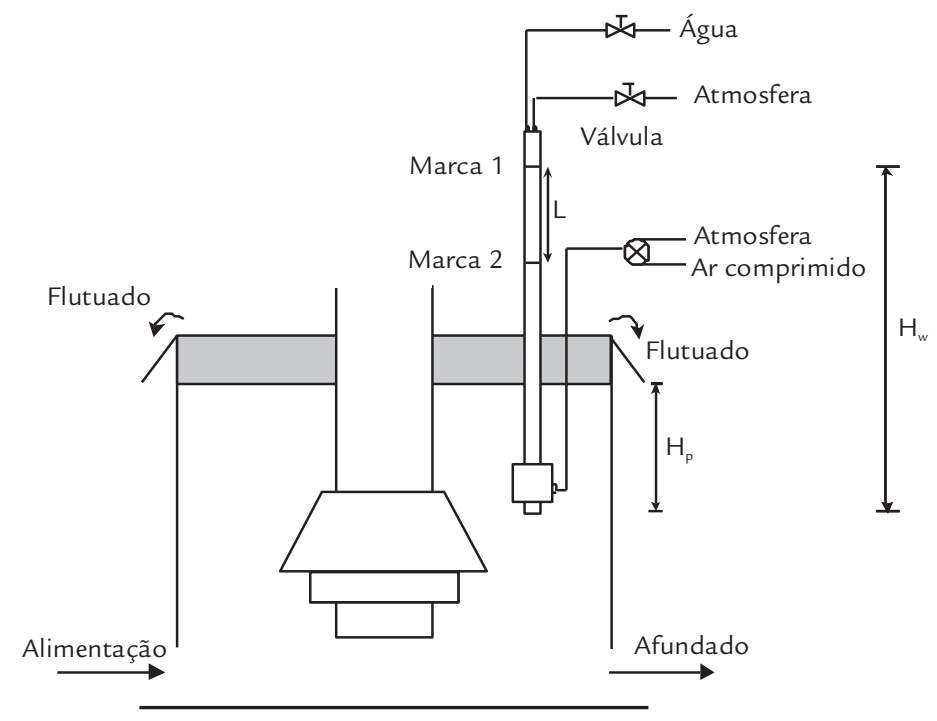




\section{Hold-up do ar}

A sonda utilizada, para determinar o hold-up local, nas células de flotação (sonda- $\varepsilon_{g}$ ), está ilustrada na Figura 2. A medida consiste, basicamente, no aprisionamento de uma fração de polpa aerada. Para tanto, a sonda- $\varepsilon_{g}$ foi colocada dentro da célula de flotação e um volume conhecido de polpa aerada foi coletado em um cilindro. Foi medido o volume de polpa

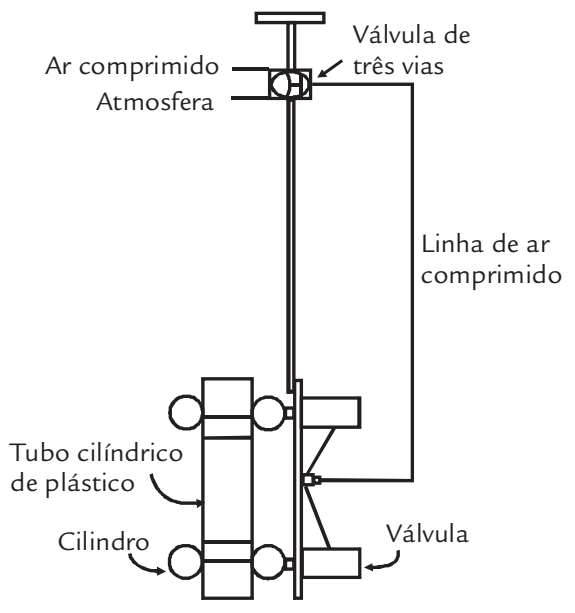

não aerada e, por diferença, foi calculado o volume de ar presente no cilindro. Aplicando-se a Eq. (4), foi calculado o hold-up local, em cada célula de flotação.
Figura 2

Sonda utilizada para medir o hold-up do ar, em célula mecânica de flotação.

\section{Determinação do tamanho médio de bolhas e fluxo de área superficial}

A determinação do tamanho médio de bolhas de ar, nos circuitos de flotação da RPM e de Taquari-Vassouras, foi feita utilizando-se uma sonda em que bolhas de ar eram trazidas do interior da polpa, por meio de um tubo cilíndrico transparente, para uma área

\section{Resultados e discussões}

A Figura 3 ilustra os parâmetros de dispersão de ar $\left(J_{g}, \varepsilon_{g}\right.$ e $\left.d_{32}\right)$, do circuito rougher e scavenger da linha 3 da planta de flotação de silvita de Taquari-Vassouras. Os resultados mostram que, em um mesmo banco de células de flotação, há uma variação nos valores dos parâmetros de dispersão de ar, sugerindo que as células de flotação não operam com o mesmo grau de eficiência. Tal observação permite ao engenheiro de processos minerais a identificação de quais equipamentos apresentam os resultados mais discrepantes e, a partir dessa observação, promover uma investigação mais detalhada das causas, podendo, então, levantar dados para corrigir esses problemas. No caso em questão, a redução, na magnitude do $J_{g}$ e do $\varepsilon_{g}$, ao longo do banco de células, pode ser devida a fatores que restrinjam a capacidade de alimentação de ar para a célula, tais como o entupimento na entrada de ar da célula ou, ainda, o desgaste das pás do impelidor. No caso específico da flotação de silvita (sais solúveis), os baixos valores plana fora da célula de flotação, em que tais bolhas poderiam ser visualizadas e, então, fotografadas.

Em seguida, por meio de um programa analisador de imagens, foi possível a caracterização da curva de distribuição de tamanho de bolhas. Foi

de $J_{g}$ também podem ser devidos à alta concentração de eletrólitos nesse tipo de sistema, como reportado por Joshi e Sharma (1977) apud Patwardhan e Joshi (1999). Na Figura 3, também pode ser observado que as duas últimas células do banco (scavenger 1 e 2) apresentam um aparente paradoxo, uma vez que há um aumento do $J_{g}$ à medida que o $\varepsilon_{g}$ diminui. Tal comportamento, no entanto, ilustra uma deficiência na hidrodinâmica do sistema, conduzindo, dessa forma, a uma distribuição irregular do ar dentro dessas células. Essa variação na magnitude do $J_{g}$ afeta a qualidade da suspensão de sólidos dentro da célula.

Também pode ser observado, na Figura 3, que o diâmetro médio de bolhas, representado pelo diâmetro médio de Sauter $\left(d_{32}\right)$, aumenta à medida que se avança no banco de células. Essa variação no tamanho médio de bolhas também ilustra que as células de flotação não estão operando de forma uniforme, conduzindo a uma dispersão ineficiente do ar dentro das células. possível, também, elaborar o cálculo do diâmetro médio de Sauter. Os detalhes da realização dessas medidas podem ser encontrados em Weedon et al. (2005).

A Tabela 1 apresenta as características das células de flotação investigadas.

Outras medições dos parâmetros de dispersão do ar em usinas brasileiras estão reunidas na Tabela 2. Observa-se que os circuitos investigados na usina de Taquari-Vassouras apresentaram valores médios semelhantes entre si, independentemente do tipo de célula de flotação (Denver vs Eimco) ou do tipo de circuito (rougher vs scavenger). A velocidade superficial do ar variou na faixa de $0,04 \mathrm{~cm} / \mathrm{s}$ a $0,06 \mathrm{~cm} / \mathrm{s}$. enquanto que o hold-up do ar ficou na faixa de 3,35$5,30 \%$. Convém salientar que tais valores são considerados baixos, quando são comparados com os valores comumente reportados na literatura. Na literatura, apesar de esses valores apresentarem-se baixos, como 3\%, para alguns tipos de células, a maioria dos resultados apresentados para o hold-up do ar está na faixa de $15 \%$ a $22 \%$ (Deglon et al., 2000). No entanto, como comentando anteriormente, trata-se de um sistema diferenciado, em que predomina uma alta concentração de eletrólitos e que deve afetar a magnitude dos parâmetros de dispersão de ar 


\begin{tabular}{|c|c|c|c|c|c|c|c|c|}
\hline Usina & Linha & $\begin{array}{c}\text { Fabricante } \\
\text { célula de } \\
\text { flotação }\end{array}$ & Circuito & $\begin{array}{c}\text { Volume } \\
\text { Nominal } \\
\left(\mathrm{m}^{3}\right)\end{array}$ & $\begin{array}{c}\text { Seção } \\
\text { Transversal } \\
\left(\mathbf{m}^{2}\right)\end{array}$ & $\begin{array}{c}\text { Potência } \\
(\mathrm{kW})\end{array}$ & \begin{tabular}{|} 
Rotação \\
do \\
Impelidor \\
(rpm)
\end{tabular} & $\begin{array}{c}\text { Altura } \\
\text { Média- } \\
\text { Camada } \\
\text { Espuma (m) }\end{array}$ \\
\hline \multirow{13}{*}{$\begin{array}{l}\text { Taquari- } \\
\text { Vassouras }\end{array}$} & \multirow{8}{*}{3} & \multirow{8}{*}{ DENVER } & Rougher-1 & 3 & 3 & n.m & 210 & 0,10 \\
\hline & & & Rougher-2 & 3 & 3 & n.m & 210 & 0,10 \\
\hline & & & Rougher-3 & 3 & 3 & n.m & 209 & 0,10 \\
\hline & & & Rougher-4 & 3 & 3 & n.m & 209 & 0,10 \\
\hline & & & Rougher-5 & 3 & 3 & n.m & 194 & 0,10 \\
\hline & & & Rougher-6 & 3 & 3 & n.m & 194 & 0,10 \\
\hline & & & Scavanger-1 & 3 & 3 & n.m & 194 & 0,10 \\
\hline & & & Scavanger-2 & 3 & 3 & n.m & 193 & 0,10 \\
\hline & \multirow{5}{*}{4} & \multirow{5}{*}{ EIMCO } & Rougher-1 & 15 & 8,5 & n.m & 110 & 0,10 \\
\hline & & & Rougher-2 & 15 & 8,5 & n.m & 122 & 0,10 \\
\hline & & & Rougher-3 & 15 & 8,5 & n.m & 109 & 0,10 \\
\hline & & & Rougher-4 & 15 & 8,5 & n.m & 120 & 0,10 \\
\hline & & & Scavanger-1 & 15 & 8,5 & n.m & 120 & 0,10 \\
\hline \multirow{4}{*}{$\begin{array}{c}\text { Rio Paracatu } \\
\text { Mineração - } \\
\text { RPM }\end{array}$} & \multirow{4}{*}{ C } & \multirow{4}{*}{ WEMCO } & Scavanger-1 & 130 & 28 & 116 & 145 & 0,25 \\
\hline & & & Scavanger-2 & 130 & 28 & 126 & 145 & 0,12 \\
\hline & & & Scavanger-3 & 130 & 28 & 114 & 145 & 0,18 \\
\hline & & & Scavanger-4 & 130 & 28 & 127 & 145 & 0,13 \\
\hline \multirow{9}{*}{$\begin{array}{c}\text { Fosfertil- } \\
\text { Catalão - } \\
\text { CMC } \\
\text { (Vale } \\
\text { Fertilizantes) }\end{array}$} & \multirow{9}{*}{$\begin{array}{l}\text { Circuito } \\
\text { Rougher }\end{array}$} & \multirow{9}{*}{ WEMCO } & Rougher-1MF1 & 14,2 & 10 & n.m & 186 & n.m \\
\hline & & & Rougher-1MF2 & 14,2 & 10 & n.m & 231 & n.m \\
\hline & & & Rougher -MF3 & 14,2 & 10 & n.m & 230 & n.m \\
\hline & & & Rougher-1MF4 & 14,2 & 10 & n.m & 229 & n.m \\
\hline & & & Rougher-2MF1 & 14,2 & 10 & n.m & 231 & n.m \\
\hline & & & Rougher-2MF2 & 14,2 & 10 & n.m & 231 & n.m \\
\hline & & & Rougher-2MF3 & 14,2 & 10 & n.m & 231 & n.m \\
\hline & & & Rougher-2MF4 & 14,2 & 10 & n.m & 231 & n.m \\
\hline & & & Rougher-2MF5 & 14,2 & 10 & n.m & 185 & n.m \\
\hline \multirow{12}{*}{ Copebras } & \multirow{6}{*}{ Usina 47} & \multirow{12}{*}{ WEMCO } & Rougher-1 & 8,5 & 7 & n.m & 222 & n.m \\
\hline & & & Rougher-2 & 8,5 & 7 & n.m & 222 & n.m \\
\hline & & & Rougher-3 & 8,5 & 7 & n.m & 222 & n.m \\
\hline & & & Rougher-4 & 8,5 & 7 & n.m & 222 & n.m \\
\hline & & & Rougher-5 & 8,5 & 7 & n.m & 222 & n.m \\
\hline & & & Rougher- 6 & 8,5 & 7 & n.m & 222 & n.m \\
\hline & \multirow{6}{*}{ Usina 76} & & Rougher-1 & 8,5 & 7 & n.m & 200 & n.m \\
\hline & & & Rougher-2 & 8,5 & 7 & n.m & 207 & n.m \\
\hline & & & Rougher-3 & 8,5 & 7 & n.m & 200 & n.m \\
\hline & & & Rougher-4 & 8,5 & 7 & n.m & 205 & n.m \\
\hline & & & Rougher-5 & 8,5 & 7 & n.m & 205 & n.m \\
\hline & & & Rougher- 6 & 8,5 & 7 & n.m & 200 & n.m \\
\hline
\end{tabular}

Características principais das células mecânicas avaliadas.

Figura 3

Parâmetros de dispersão de ar em circuito de células mecânicas de flotação (Denver) de Taquari-Vassouras.

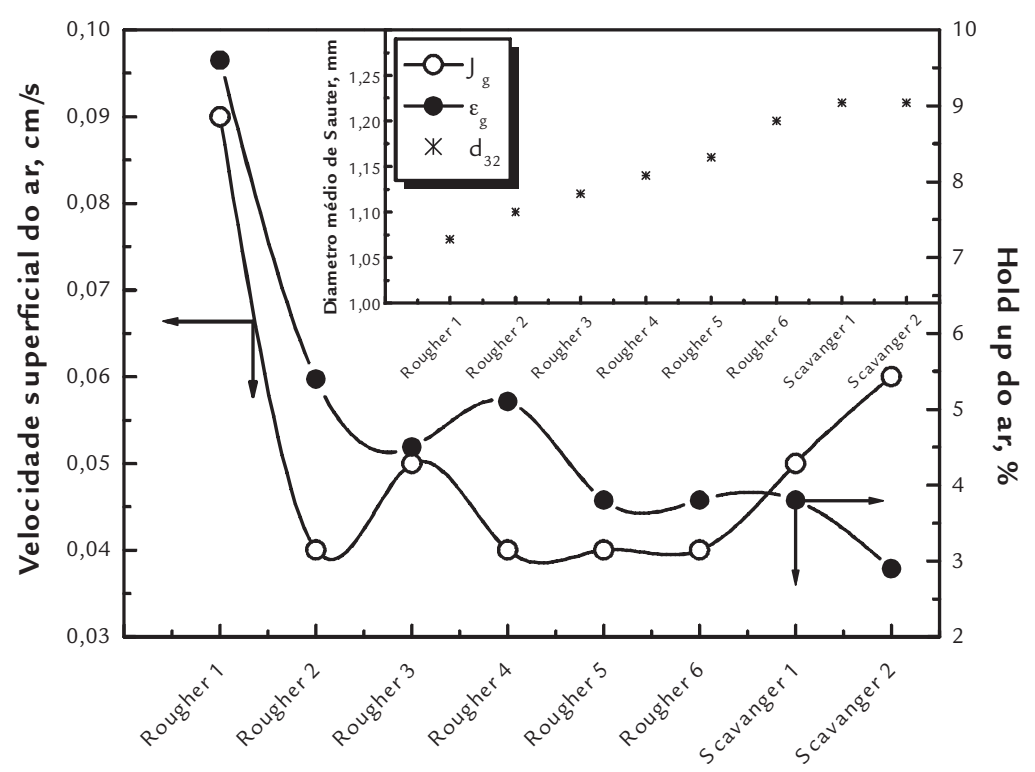

REM: R. Esc. Minas, Ouro Preto, 65(2), 271-277, abr. jun. | 2012275 
sem, necessariamente, comprometer o desempenho do processo.

A maior discrepância foi apresentada pelo circuito de flotação da RPM, que apresentou os maiores valores de hold-up do ar, em torno de 31\%, e também de tamanho médio de bolhas, $2,61 \mathrm{~mm}$. Tais valores são considerados elevados, mesmo quando tais valores são comparados com aqueles reportados na literatura (Deglon et al., 2000; Gorain et al., 1995; Schwarz e Alexander., 2005). As indústrias de beneficiamento de rocha fosfática Copebrás e Fosfértil (atual Vale Fertilizantes) apresentaram resultados que, apesar de diferirem um pouco entre si, podese considerar que estão na mesma faixa

\begin{tabular}{|c|c|c|c|c|c|c|c|c|}
\hline \multirow[t]{2}{*}{ Usina e Circuito } & \multicolumn{2}{|c|}{$\begin{array}{c}\text { Velocidade } \\
\text { Superficial do ar } \\
J_{g}(\mathrm{~cm} / \mathrm{s})\end{array}$} & \multicolumn{2}{|c|}{$\begin{array}{l}\text { Hold-up do ar } \\
\qquad \varepsilon_{\mathrm{g}}(\%)\end{array}$} & \multicolumn{2}{|c|}{$\begin{array}{c}\text { Diâmetro médio } \\
\text { de Sauter } \\
d_{32}(\mathrm{~mm})\end{array}$} & \multicolumn{2}{|c|}{$\begin{array}{c}\text { Fluxo da área } \\
\text { superficial das bolhas } \\
\mathrm{S}_{\mathrm{b}}\left(\mathrm{s}^{-1}\right)\end{array}$} \\
\hline & Média & $\begin{array}{l}\text { Desvio- } \\
\text { Padrão }\end{array}$ & Média & $\begin{array}{l}\text { Desvio- } \\
\text { Padrão }\end{array}$ & Média & $\begin{array}{l}\text { Desvio- } \\
\text { Padrão }\end{array}$ & Média & $\begin{array}{l}\text { Desvio- } \\
\text { Padrão }\end{array}$ \\
\hline $\begin{array}{l}\text { Taquari- Vassouras } \\
\text { /Rougher (Denver) }\end{array}$ & 0,05 & 0,02 & 5,37 & 2,18 & 1,13 & 0,05 & 2,63 & 1,08 \\
\hline $\begin{array}{c}\text { Taquari - } \\
\text { Vassouras / } \\
\text { Scavanger (Denver) }\end{array}$ & 0,06 & 0,01 & 3,35 & 0,64 & 1,22 & 0,00 & 2,80 & 0,14 \\
\hline $\begin{array}{c}\text { Taquari-Vassouras / } \\
\text { Rougher (Eimco) }\end{array}$ & 0,05 & 0,01 & 5,08 & 0,47 & 1,28 & 0,42 & 2,40 & 0,59 \\
\hline $\begin{array}{c}\text { Taquari-Vassouras } \\
\text { Scavanger (Eimco) }\end{array}$ & 0,04 & 0,01 & 5,30 & 0,87 & 1,19 & 0,00 & 2,80 & 0,00 \\
\hline $\begin{array}{c}\text { RPM / Scavanger } \\
\text { (Wemco) }\end{array}$ & 0,88 & 0,18 & 31,50 & 10,61 & 2,61 & 0,40 & 20,05 & 3,61 \\
\hline $\begin{array}{c}\text { CMC / Rougher } 1 \\
\text { (Wemco) }\end{array}$ & 0,71 & 0,17 & 7,71 & 1,17 & n.m & n.m & n.m & n.m \\
\hline $\begin{array}{c}\text { CMC / Rougher } 2 \\
\text { (Wemco) }\end{array}$ & 0,52 & 0,06 & 6,09 & 0,90 & n.m & n.m & n.m & n.m \\
\hline $\begin{array}{c}\text { Copebras / } \\
\text { Rougher Unidade } \\
47 \text { (Wemco) }\end{array}$ & 0,90 & 0,14 & 8,50 & 0,84 & $\mathrm{n} . \mathrm{m}$ & n.m & n.m & n.m \\
\hline $\begin{array}{c}\text { Copebras / } \\
\text { Rougher Unidade } \\
76 \text { (Wemco) }\end{array}$ & 1,17 & 0,16 & 6,98 & 1,64 & n.m & n.m & n.m & n.m \\
\hline
\end{tabular}

\section{Conclusões}

Os parâmetros de dispersão de ar (velocidade superficial do ar, hold-up do ar, tamanho de bolhas e fluxo de área superficial de bolhas) de algumas usinas brasileiras de beneficiamento de minério foram medidos, experimentalmente, e apresentados com o intuito de contribuir para um melhor entendimento dos processos envolvidos com a hidrodinâmica de circuito de flotação de minérios em usinas brasileiras. Foi observado que, com exceção da RPM, os circuitos investigados apresentaram baixos valores de $J_{g}, \varepsilon_{g}$ e $d_{32}$. Os resultados, também, sugerem uma distribuição irregular do ar dentro das células investigadas, haja vista a obtenção de valores incoerentes dos parâmetros de dispersão de ar para um mesmo equipamento avaliado. Foram encontrados valores para o fluxo de área superficial de bolhas, $S_{b} \leq 20 \mathrm{~s}^{-1}$, consideravelmente menores do que aqueles reportados na literatura, para de valores. O fluxo de área superficial de bolhas foi medido somente para as usinas de Taquari-Vassouras e RPM. Os resultados encontrados, para os circuitos investigados dessas duas usinas, estão abaixo daqueles reportados pela literatura, para células autoaeradas, em que $S_{b}$ está na faixa de 30-165 $\mathrm{s}^{-1}$ (Deglon et al., 2000; Gorain et al., 1996).
Tabela 2

Parâmetros de dispersão

de ar em usinas brasileiras.

\section{Agradecimentos}

Os autores agradecem às empresas RPM, Copebras e Vale Fertilizantes pela permissão para a execução dos trabalhos e a consequente publicação dos resultados.

À CAPES pelo apoio financeiro.

Aos integrantes do projeto AMIRA P260D - David Weedon células autoaeradas $\left(40 \leq S_{b} \leq 165 \mathrm{~s}^{-1}\right)$. A escassez de dados hidrodinâmicos nas usinas brasileiras apontam para o fato de que pouco tem sido feito para medir tais variáveis em usinas nacionais. Faz-se, então, necessário construir um diagnóstico de desempenho de células de flotação que tenha como base, além dos fatores físico-químicos, também fatores hidrodinâmicos. Assim, uma avaliação mais completa poderá, futuramente, ser realizada. e Tim Akroyd pelo trabalho conjunto na realização das medidas nos circuitos da RPM e TaquariVassouras. 


\section{Referências bibliográficas}

AHMED, N., JAMESON, G. J. Flotation Kinetics. Mineral Processing Extr. Metal Review, v. 5. p. 77-99, 1989.

CILEK, E. C., YILMAZER, B. Z. Effect of hydrodynamic parameters on entrainment and flotation performance. Mineral Engineering, v. 16, p. 745-756, 2003.

DEGLON, D. A., EGYA-MENSAH, D., FRANZIDIS, J. P. Review of hydrodynamics and gas dispersion in flotation cells on South African platinum concentrators. Mineral Engineering, v. 13, n. 3, p. 235-244, 2000.

GORAIN, B. K., FRANZIDIS, J. P., MANLAPIG, E. V. Studies on impeller type, impeller speed and air flow rate in an industrial scale flotation cell - Part 1: Effect on bubble size distribution. Minerals Engineering, v. 8 n.6, p. 615-635, 1995.

GORAIN, B. K., FRANZIDIS, J. P., MANLAPIG, E. V. Studies on impeller type, impeller speed and air flow rate in an industrial scale flotation cell Part 2: Effect on gas hold-up. Minerals Engineering, v. 8, n. 12, p. 1557-1570, 1995.

GORAIN, B. K., FRANZIDIS, J. P., MANLAPIG, E. V. Studies on impeller type, impeller speed and air flow rate in industrial scale flotation cell. Part 3: Effect on superficial gas velocity. Minerals Engineering, v. 9, n. 6, p. 639-654, 1996.

GRAU, R. A., HEISKANEN, K. Bubble size distribution in laboratory scale flotation cells. Minerals Engineering, v. 18, p. 1164-1172, 2005.

JOSHI, J. B., SHARMA, M. M. Mass Transfer and Hydrodynamic Characteristics of Gas Inducing Type of Agitated Contactors. The Canadian Journal of Chemical Engineering, v. 55 (6), p. 683-695, 1977.

LEAL FILHO, L. S., LIMA, O. A. Caracterização Hidrodinâmica de Condicionadores de Reagentes e Células Mecânicas de Flotação da Ultrafértil S/A, Complexo de Mineração de Catalão (CMC). Relatório Confidencial. LFQI/PMI/EPUSP, 112p. 2010.

LIMA, O. A. Suspensão de partículas grossas em células mecânicas de flotação. São Paulo: Programa de Pós-graduação em Engenharia Mineral da EPUSP, 2009. 231p. (Tese de Doutorado).

LIMA, O. A., LEAL FILHO, L. S., SILVA, A. L., MOURA, A. J. Distribuição de tempos de Residência em Células Mecânicas de Flotação. REM - Revista Escola de Minas, v. 58, n. 3, p. 213 -218, 2005.

PATWARDHAN, A.W., JOSHI, J. B. Design of gas-including reactors. Industrial and Engineering Chemistry Research, v. 38, p.49-80, 1999.

SCHWARZ, S., ALEXANDER, D. Gas dispersion measurements in industrial flotation cells. Minerals Engineering, v. 19, p. 554-560, 2006.

VILLANUEVA, F., WEBER, A., PRADO, S. A maior célula de flotação do mundo. Brasil Mineral, n. 242, p. 163-165, 2005.

VERA, M. A touch of froth: how bubble-particles aggregates take the strain. University of Queensland, 2002. (PhD Thesis).

WEBER, A., WALKER, C., REDDEN, L. Scale up and design of large-scale flotation equipment. In: Advances in Flotation Technology, Eds. PAREKH, B.K., MILLER, J. D. SME Inc. Pub., USA, p. 353-369, 1999.

WEEDON, D., ZANIN, M., SKINNER, B., AKROYD, T., LEAL FILHO, L. S. Report on site study at CVRD's Flotation Plant of Taquari-Vassouras. Ian Wark Institute, Project P260D (AMIRA), 2005.

YIANATOS, J., BERGH, L., CONDORI, P., AGUILERA, J. Hydrodynamic and metallurgical characterization of industrial flotation banks for control purposes. Minerals Engineering, v. 14, n. 9, p. 1033-1046, 2001.

Artigo recebido em 31 de julho de 2011. Aprovado em 07 de novembro de 2011. 